\title{
Decision-making for flexible manufacturing systems using DEMATEL and SAW
}

\author{
Reza Talebanpour* and Mehrdad Javadi
}

Department of Industrial Engineering, Islamic Azad University, South Tehran Branch, Tehran, Iran

\begin{tabular}{l}
\hline C H R O N I C L E \\
\hline Article history: \\
Received December 10, 2014 \\
Received in revised format: \\
March 2, 2015 \\
Accepted March 28, 2015 \\
Available online \\
April 11 2015 \\
\hline Keywords: \\
Decision making \\
DEMATEL \\
Flexible manufacturing system \\
Simple Additive Weighting \\
\hline
\end{tabular}
\begin{abstract}
A B S T R A C T
Flexible manufacturing system (FMS) is an important component of competitive strategy, which could be used for improving organizational performance, productivity, and profitability. The goal of this research is to use DEMATEL approach for finding the intensity of influence of selected criteria. Then, in order to evaluate flexible manufacturing systems, the results of DEMATEL are used in SAW method. A questionnaire was developed and ten professional experts working in various departments of Aluminum Composite Panel Industry are asked to answer its questions. The obtained results reveal that in this case, it is a better choice not to implement and develop FMS.
\end{abstract}

\section{Introduction}

Over the last several decades, flexible manufacturing system (FMS) has been a subject of intense research. Therefore, many papers have been published on this subject (Bayazit, 2005). The aim of designing an FSM is to combine the flexibility of a job shop and the efficiency of a mass production line, so that various work pieces could be produced on a group of machines (Chan et al., 1997). According to Matte et al. (2001), FMSs were introduced in the late twentieth century as a highly competitive manufacturing strategy, and they have been in the center of attention of many researchers. An FMS uses computer controlled automation systems to integrate the material handling system (MHS) with the machine centers (MCs). It has been widely used in shop floors for producing a large set of product families in small or medium volumes. Another definition presented by Solimanpur et al. (2005) describes flexible manufacturing system (FSM) as "a production system in which a central computer links and controls a set of machines and a flexible material-handling system such as an automated guided vehicle (AGV), a robot, etc.”

\footnotetext{
* Corresponding author

E-mail address: St_r_talebanpour@azad.ac.ir (R. Talebanpour) 
The FMS is called 'flexible' because it is able to simultaneously process a wide range of various part styles at different workstations, and it can respond to chang demand patterns by adjusting the mix of part styles and quantities of production. The evolution of FMSs provides great potential for increasing flexibility and changing the basis of competition by ensuring both customized and cost-effective manufacturing at the same time (Rao, 2007).

So far, the potential benefits of FMS implementation have been the subject of many studies. All these studies have reached a common conclusion that the FMS implementation has numerous advantages. The advantages of a successful FMS implementation include increased flexibility and product variety, enhanced responsiveness and productivity, decreased labor costs, and increased machinery utilization (Inman, 1991; Boer \& Krabbendam, 1991, 1992; Evans \& Haddock, 1992; Kaighobadi \& Venkatesh, 1994; Maffei and Meredith, 1995; Bayazit, 2005). Several multi criteria decision making (MCDM) approaches have been used in existing literature to determine the relative importance of a set of features or criteria. Simple Additive Weighting (SAW) method is one of these techniques designed for solving complicated multi-criteria problems. A large number of quantitative techniques have attempted to evaluate new technology implementation. Previously, Shang and Sueyoshi (1995), Myint and Tabucanon (1994), Albayrakoglu (1996), Shamsuzzaman et al. (2003) and Chan et al. (2000), Bayazit (2005) have used another technique known as analytical hierarchy process (AHP) to evaluate advanced technology.

To evaluate FMS, this paper presents a comprehensive application of Decision Making Trial and Evaluation Laboratory (DEMATEL) and SAW techniques for a real-world case. This study is aimed at determining whether or not ZARIN BOND (ZB) should implement FMS throughout the plant by using the DEMATEL and SAW. This paper is organized as follows: The first section presents the research carried out at ZB. The attributes affecting the decision are presented in the second section. In the third section, the DEMATEL is used to specify the weight of the criteria at ZB. The SAW method and its application in selecting the best alternative are presented in the fourth section. Finally, after the matrix calculation section with future scope of further research, the last section concludes the paper.

\section{Research methodology}

ZARIN BOND (ZB) was founded in 2009 in Shiraz, the capital city of Fars province, in Iran. It is now the major manufacturer of Aluminum Composite Panels in the country. Through the last three years, it has intensively invested to obtain the most modern technologies e.g. computer numerical control (CNC) machines and FMSs. The management of ZB is now considering the implementation of FMS throughout the organization. By developing an FMS they gained significant advantages such as increasing flexibility, decreasing set-up time, and increasing customer satisfaction, etc. However, they also encountered some problems during the implementation of FMS. Thus, they were eager to find out that whether or not the FMS should be developed in entire plant. In order to provide a systematic approach, we ran a study based on DEMATEL and SAW. We visited the company managers and it took several hours to decide on the best alternative. A team was formed from the decision makers of ZB. This team consisted of production manager, operations manager, quality control manager, and sales manager. Since the members of decision making team were not familiar with the approach, as the first step, the DEMATEL methodology was introduced to them. Next, the model was formulated and the criteria were determined. 17 factors were considered as primary ones. Also, we identified two different alternatives:

(1) Developing FMS; and

(2) Not developing FMS

Once the criteria were determined, the decision-making team performed pair wise comparisons including all possible combinations of criteria. Since the decision concerned implementation of FMS 
in the entire plant, decision makers used their own experience on partial FMS implementation to determine the criteria. The criteria for evaluating the decision were determined and used in the DEMATEL approach. These criteria are shown in Table 1. Next, in order to evaluate flexible manufacturing systems and select the best alternative, the results of DEMATEL were used in SAW approach.

\section{Table 1}

The selected criteria

\begin{tabular}{llll}
\hline Item & Description & Item & Description \\
\hline 1 & quality improvement & 10 & long-term competitive power \\
2 & faster delivery & 11 & training employees \\
3 & product variety & 12 & high initial costs \\
4 & customer satisfaction & 13 & necessity of developing company \\
5 & set-up time & 14 & space requirements \\
6 & production time & 15 & long implementation lead-time \\
7 & labor cost & 16 & labor requirements \\
8 & productivity & 17 & central computer control \\
9 & machine utilization & & \\
\hline
\end{tabular}

\subsection{DEMATEL Method}

Decision Making Trial and Evaluation Laboratory (DEMATEL) is a comprehensive technique used to create and analyze a structural model of the causal relationships between numerous and complicated factors (Lin \& Lin, 2008). This approach was used for the first time at Battelle Memorial Institute in Geneva between 1972 and 1976 for solving a group of complicated and intertwined problems (Tzeng \& Huang, 2011; Mehregan et al., 2012). This technique is able to improve the understanding of the particular problematic group of interacted factors and criteria also; by constructing a hierarchical relevant network system, it can provide a feasible solution. It is a widely accepted idea that DEMATEL is one of the best tools, which could be used for solving the cause and effect relationship between the evaluation criteria (Chiu et al., 2006, Liou et al., 2007, Tzeng et al., 2007, Wu and Lee, 2007, Lin and Tzeng, 2009). The DEMATEL technique is used for analyzing and constructing the cause and effect relationship between evaluation criteria (Yang et al., 2008) or for extracting interrelationship between factors (Lin and Tzeng, 2009). According to Yu and Tseng (2006), Liou, et al., (2007), Tzeng, et al., (2007), Yang, et al., (2008), Wu and Lee (2007), Shieh et al., (2010), this methodology is capable of confirming interrelations between variables/features, and it is also able to limit the relations that reflect the characteristics of a system (Amiri et al., 2011).

The final output of DEMATEL technique is a visual representation known as mind map. Based on this visual representation, the respondent organizes their actions regarding the world (Amiri et al., 2011; Lin \& Lin, 2008) or adheres to the priorities expressed in it (Tzeng \& Huang, 2011; Mehregan et al., 2012).

\subsubsection{Steps of DEMATEL method include}

\section{Finding the average matrix}

In this study, we deal with $H$ experts (ten people) and $n$ factors (seventeen factors). Each expert is requested: "To what degree does factor $i$ affect factor $j$ ?" these pair wise comparisons between all the factors are made and are represented with $a_{i j}$. It is noteworthy that integers 0-4 are ascribed to these comparisons (Tzeng et al., 2007). Among which zero indicates "no influence" of $i$ factor on $j$ factor, one represents "very low influence", two indicates "medium/low influence", three represents "high 
influence" and finally four shows "very high influence". Then, by calculating the formula below, we can calculate mean $A_{n \times n}$ matrix (Lin \& Lin, 2008) for all the experts' opinions by obtaining the mean of $\mathrm{H}$ scores as follows:

$$
a_{i j}=\frac{1}{H} \sum_{k=1}^{H} x_{i j}^{k}
$$

It is noteworthy that the average matrix $\mathrm{A}=\left[a_{i j}\right]$ is also called initial direct relation matrix (Lin \& Lin, 2008).

2. Calculating the normalized initial direct-relation matrix

We turn matrix $A$ into normalized initial direct-relation matrix called matrix $D$ as follows,

$$
m=\min \left[\frac{1}{\max \sum_{j=1}^{n}\left|a_{i j}\right|}, \frac{1}{\max \sum_{i=1}^{n}\left|a_{i j}\right|}\right]
$$

And then

$$
\mathrm{D}=\mathrm{M} \times \mathrm{A}
$$

\begin{tabular}{|c|c|c|c|c|c|c|c|c|c|c|c|c|c|c|c|c|c|}
\hline Criteria & 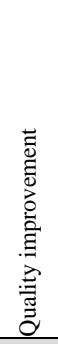 & 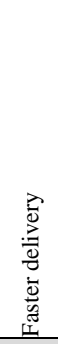 & 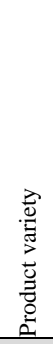 & 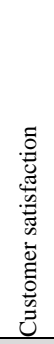 & 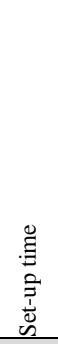 & 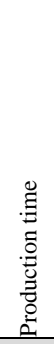 & 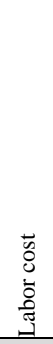 & 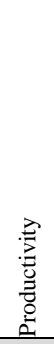 & 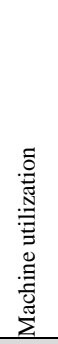 & 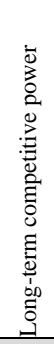 & 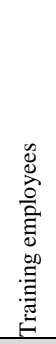 & 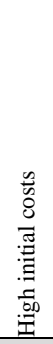 & 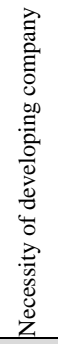 & 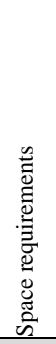 & 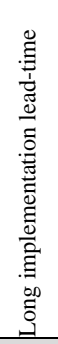 & 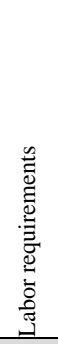 & 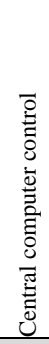 \\
\hline Quality improvement & 0.00 & 3.25 & 3.51 & 3.74 & 3.79 & 2.89 & 3.68 & 3.85 & 2.94 & 3.91 & 2.99 & 3.76 & 3.89 & 2.87 & 3.84 & 3.47 & 3.95 \\
\hline Faster delivery & 0.97 & 0.00 & 0.83 & 3.87 & 0.78 & 0.93 & 0.98 & 3.28 & 2.21 & 3.39 & 1.67 & 3.32 & 3.34 & 2.12 & 2.77 & 1.35 & 2.43 \\
\hline Product variety & 3.57 & 3.74 & 0.00 & 2.81 & 3.17 & 3.29 & 1.54 & 3.32 & 2.53 & 3.34 & 3.97 & 3.87 & 3.20 & 3.94 & 3.62 & 3.10 & 1.98 \\
\hline Customer satisfaction & 0.88 & 1.37 & 1.24 & 0.00 & 0.78 & 0.67 & 0.68 & 0.90 & 1.38 & 3.77 & 0.87 & 0.89 & 3.93 & 0.75 & 3.10 & 2.62 & 1.39 \\
\hline Set-up time & 1.29 & 2.81 & 0.93 & 0.97 & 0.00 & 0.95 & 3.51 & 2.64 & 1.26 & 2.37 & 1.63 & 1.54 & 1.74 & 0.24 & 0.34 & 1.62 & 1.21 \\
\hline Production time & 3.83 & 3.75 & 2.10 & 2.14 & 0.87 & 0.00 & 2.48 & 3.64 & 0.98 & 3.37 & 1.96 & 3.86 & 3.83 & 0.61 & 0.88 & 0.39 & 2.62 \\
\hline Labor cost & 2.99 & 3.13 & 2.24 & 1.20 & 2.44 & 2.97 & 0.00 & 2.81 & 0.82 & 3.36 & 1.81 & 2.57 & 3.35 & 0.92 & 0.79 & 3.41 & 0.86 \\
\hline Productivity & 1.64 & 2.26 & 2.11 & 1.28 & 1.16 & 0.99 & 2.86 & 0.00 & 2.94 & 3.56 & 2.11 & 1.79 & 3.09 & 0.67 & 2.09 & 2.86 & 1.20 \\
\hline Machine utilization & 3.88 & 3.77 & 3.48 & 2.55 & 1.04 & 3.55 & 3.56 & 3.74 & 0.00 & 3.96 & 2.41 & 3.34 & 3.57 & 3.10 & 3.17 & 3.60 & 1.13 \\
\hline Long-term competitive power & 0.89 & 1.36 & 0.85 & 0.85 & 0.96 & 0.83 & 1.15 & 1.35 & 0.75 & 0.00 & 1.29 & 1.13 & 0.95 & 0.79 & 1.22 & 0.55 & 0.88 \\
\hline Training employees & 3.49 & 3.66 & 1.77 & 0.88 & 2.56 & 3.75 & 1.54 & 2.56 & 0.85 & 0.92 & 0.00 & 1.15 & 2.21 & 1.56 & 0.70 & 2.50 & 0.03 \\
\hline High initial costs & 3.66 & 3.87 & 3.66 & 1.88 & 2.09 & 3.98 & 3.66 & 3.96 & 2.09 & 3.56 & 0.68 & 0.00 & 3.03 & 2.69 & 0.68 & 1.45 & 0.36 \\
\hline Necessity of developing company & 0.95 & 0.82 & 2.11 & 1.46 & 1.13 & 0.68 & 2.79 & 0.73 & 0.71 & 3.44 & 2.54 & 2.61 & 0.00 & 3.86 & 3.76 & 2.52 & 2.17 \\
\hline Space requirements & 2.15 & 2.79 & 1.18 & 0.64 & 0.57 & 0.76 & 1.10 & 0.87 & 2.62 & 1.40 & 0.94 & 0.68 & 2.10 & 0.00 & 2.40 & 1.74 & 0.52 \\
\hline Long implementation lead-time & 2.24 & 2.26 & 1.76 & 2.11 & 0.87 & 1.04 & 2.40 & 3.08 & 1.33 & 2.91 & 0.82 & 3.35 & 3.09 & 0.86 & 0.00 & 0.92 & 0.84 \\
\hline Labor requirements & 3.34 & 3.54 & 1.08 & 2.17 & 2.94 & 3.27 & 3.54 & 3.84 & 2.61 & 3.59 & 3.98 & 3.44 & 3.03 & 0.94 & 3.33 & 0.00 & 3.11 \\
\hline Central computer control & 3.88 & 2.84 & 0.67 & 2.98 & 3.75 & 3.56 & 3.47 & 3.80 & 2.84 & 3.84 & 3.27 & 3.68 & 2.55 & 2.98 & 2.94 & 2.72 & 0.00 \\
\hline
\end{tabular}

Table 2

Average matrix (initial direct relation matrix) 
Table 3 shows matrix D:

\section{Table 3}

Normalized initial direct-relation matrix (matrix D)

\begin{tabular}{lllllllllllllllll}
\hline 0.000 & 0.055 & 0.060 & 0.064 & 0.064 & 0.049 & 0.063 & 0.066 & 0.050 & 0.066 & 0.051 & 0.064 & 0.066 & 0.049 & 0.065 & 0.059 & 0.067 \\
0.016 & 0.000 & 0.014 & 0.066 & 0.013 & 0.016 & 0.017 & 0.056 & 0.038 & 0.058 & 0.028 & 0.057 & 0.057 & 0.036 & 0.047 & 0.023 & 0.041 \\
0.061 & 0.064 & 0.000 & 0.048 & 0.054 & 0.056 & 0.026 & 0.056 & 0.043 & 0.057 & 0.067 & 0.066 & 0.054 & 0.067 & 0.062 & 0.053 & 0.034 \\
0.015 & 0.023 & 0.021 & 0.000 & 0.013 & 0.011 & 0.012 & 0.015 & 0.023 & 0.064 & 0.015 & 0.015 & 0.067 & 0.013 & 0.053 & 0.045 & 0.024 \\
0.022 & 0.048 & 0.016 & 0.016 & 0.000 & 0.016 & 0.060 & 0.045 & 0.021 & 0.040 & 0.028 & 0.026 & 0.030 & 0.004 & 0.006 & 0.028 & 0.021 \\
0.065 & 0.064 & 0.036 & 0.036 & 0.015 & 0.000 & 0.042 & 0.062 & 0.017 & 0.057 & 0.033 & 0.066 & 0.065 & 0.010 & 0.015 & 0.007 & 0.045 \\
0.051 & 0.053 & 0.038 & 0.020 & 0.041 & 0.050 & 0.000 & 0.048 & 0.014 & 0.057 & 0.031 & 0.044 & 0.057 & 0.016 & 0.013 & 0.058 & 0.015 \\
0.028 & 0.038 & 0.036 & 0.022 & 0.020 & 0.017 & 0.049 & 0.000 & 0.050 & 0.060 & 0.036 & 0.030 & 0.052 & 0.011 & 0.036 & 0.049 & 0.020 \\
0.066 & 0.064 & 0.059 & 0.043 & 0.018 & 0.060 & 0.061 & 0.064 & 0.000 & 0.067 & 0.041 & 0.057 & 0.061 & 0.053 & 0.054 & 0.061 & 0.019 \\
0.015 & 0.023 & 0.014 & 0.014 & 0.016 & 0.014 & 0.020 & 0.023 & 0.013 & 0.000 & 0.022 & 0.019 & 0.016 & 0.013 & 0.021 & 0.009 & 0.015 \\
0.059 & 0.062 & 0.030 & 0.015 & 0.044 & 0.064 & 0.026 & 0.044 & 0.014 & 0.016 & 0.000 & 0.020 & 0.037 & 0.026 & 0.012 & 0.042 & 0.001 \\
0.062 & 0.066 & 0.062 & 0.032 & 0.036 & 0.068 & 0.062 & 0.067 & 0.036 & 0.061 & 0.012 & 0.000 & 0.052 & 0.046 & 0.012 & 0.025 & 0.006 \\
0.016 & 0.014 & 0.036 & 0.025 & 0.019 & 0.012 & 0.047 & 0.012 & 0.012 & 0.059 & 0.043 & 0.044 & 0.000 & 0.066 & 0.064 & 0.043 & 0.037 \\
0.037 & 0.047 & 0.020 & 0.011 & 0.010 & 0.013 & 0.019 & 0.015 & 0.045 & 0.024 & 0.016 & 0.012 & 0.036 & 0.000 & 0.041 & 0.030 & 0.009 \\
0.038 & 0.038 & 0.030 & 0.036 & 0.015 & 0.018 & 0.041 & 0.052 & 0.023 & 0.049 & 0.014 & 0.057 & 0.053 & 0.015 & 0.000 & 0.016 & 0.014 \\
0.057 & 0.060 & 0.018 & 0.037 & 0.050 & 0.056 & 0.060 & 0.065 & 0.044 & 0.061 & 0.068 & 0.058 & 0.052 & 0.016 & 0.057 & 0.000 & 0.053 \\
0.066 & 0.048 & 0.011 & 0.051 & 0.064 & 0.061 & 0.059 & 0.065 & 0.048 & 0.065 & 0.056 & 0.063 & 0.043 & 0.051 & 0.050 & 0.046 & 0.000 \\
\hline
\end{tabular}

\section{Calculating the Indirect Influence Matrix}

Indirect influence matrix is obtained as follows:

$$
I D=\sum_{i=2}^{\infty} D^{i}=D^{2}(I-D)^{-1}
$$

\section{Table 4}

Indirect influence matrix

\begin{tabular}{|c|c|c|c|c|c|c|c|c|c|c|c|c|c|c|c|c|}
\hline 0.093 & 0.104 & 0.070 & 0.073 & 0.067 & 0.079 & 0.091 & 0.102 & 0.068 & 0.120 & 0.077 & 0.096 & 0.109 & 0.068 & 0.083 & 0.081 & 0.058 \\
\hline 0.054 & 0.062 & 0.044 & 0.042 & 0.040 & 0.047 & 0.056 & 0.059 & 0.040 & 0.071 & 0.045 & 0.055 & 0.065 & 0.041 & 0.051 & 0.049 & 0.034 \\
\hline 0.081 & 0.094 & 0.066 & 0.067 & 0.060 & 0.070 & 0.084 & 0.093 & 0.062 & 107 & 0.068 & 0.085 & 0.099 & 0.060 & 0.075 & 0.072 & 0.054 \\
\hline 0.040 & 0.045 & 0.031 & 0.034 & 0.030 & 0.035 & 0.042 & 0.046 & 0.030 & 052 & 0.036 & .044 & 0.046 & .032 & 0.038 & .035 & 0.027 \\
\hline 0.041 & 0.047 & 0.033 & 0.035 & 0.032 & 0.037 & 0.040 & 0.047 & 0.031 & 0.055 & 0.036 & 0.044 & 0.050 & 0.032 & 0.039 & 0.038 & 0.028 \\
\hline 0.059 & 0.069 & 0.050 & 0.053 & 0.048 & 0.055 & 0.062 & 0.069 & 0.048 & 0.082 & 0.053 & 0.065 & 0.074 & 0.051 & 0.060 & 0.058 & 0.041 \\
\hline 0.057 & 0.067 & 0.046 & 0.050 & 0.044 & 0.050 & 0.062 & 0.067 & 0.045 & 0.078 & 0.052 & 0.063 & 0.070 & 0.046 & 0.056 & 0.051 & 0.042 \\
\hline 0.055 & 0.062 & 0.043 & 0.045 & 0.041 & 0.049 & 0.054 & 0.063 & 0.039 & 0.071 & 0.047 & 0.059 & 0.064 & 0.043 & 0.051 & 0.048 & 0.036 \\
\hline 0.080 & 0.093 & 0.064 & 0.068 & 0.062 & 0.069 & 0.081 & 0.092 & 0.063 & 0.107 & 0.070 & 0.087 & 0.099 & 0.062 & 0.076 & 0.072 & 0.056 \\
\hline 0.027 & 0.030 & 0.021 & 0.022 & 0.020 & 0.023 & 0.027 & 0.030 & 0.020 & 0.035 & 0.022 & 0.028 & 0.032 & 0.020 & 0.024 & 0.024 & 0.017 \\
\hline 0.049 & 0.058 & 0.040 & 0.044 & 0.037 & 0.042 & 0.052 & 0.059 & 0.040 & 0.069 & 0.046 & 0.057 & 0.063 & 0.038 & 0.049 & 0.045 & 0.038 \\
\hline 0.065 & 76 & 053 & 057 & 0.050 & 0.055 & 0.066 & 0.075 & 0.051 & 0.090 & 0.060 & 0.074 & 0.083 & 0.051 & 0.066 & 0.062 & 0.048 \\
\hline 0.054 & 0.061 & 0.039 & 0.041 & 0.039 & 0.047 & 0.050 & 0.059 & 0.040 & 0.065 & 0.042 & 0.053 & 0.062 & 0.037 & 0.045 & 0.045 & 0.031 \\
\hline 0.038 & 0.043 & 0.031 & 0.034 & 0.029 & 0.034 & 0.039 & 0.045 & 0.028 & 0.052 & 0.034 & 0.043 & 0.047 & 0.031 & 0.037 & 0.035 & 0.027 \\
\hline 0.047 & 0.055 & 0.040 & .040 & 0.037 & 0.043 & 0.049 & 0.054 & 0.037 & .065 & 0.042 & 0.050 & 0.059 & 39 & 0.047 & 0.046 & 0.033 \\
\hline 0.077 & 0.089 & 0.063 & 0.065 & 0.058 & 0.067 & 0.079 & 0.089 & 0.057 & 0.102 & 0.064 & 0.082 & 0.094 & 0.060 & 0.070 & 0.071 & 0.050 \\
\hline 0.078 & 0.092 & 0.066 & 0.065 & 0.058 & 0.068 & 0.081 & 0.090 & 0.059 & 0.105 & 0.066 & 0.083 & 0.097 & 0.058 & 0.072 & 0.071 & 0.054 \\
\hline
\end{tabular}

\section{Sequence of occurrence of elements is met}

To this end, in this step we determine the possible hierarchy or structure of the elements. The order of influence of presumed elements of one problem on other elements or their being influenced is definitely indicative of the possible structure of the hierarchy of those elements in improving or solving the problem. Therefore, in Table 5 which represents the sequence of elements (hierarchy) we use $D(I-D)^{-1}$ matrix (Mehregan et al., 2012). 
Table 5

Matrix D (I-D) ${ }^{-1}$

\begin{tabular}{|c|c|c|c|c|c|c|c|c|c|c|c|c|c|c|c|c|c|}
\hline 0.093 & 0.159 & 0.129 & 0.137 & 0.131 & 0.128 & 0.154 & 0.168 & 0.118 & 0.186 & 0.128 & 0.159 & 0.175 & 0.117 & 0.148 & 0.140 & 0.125 & 2.397 \\
\hline 0.071 & 0.062 & 0.058 & 0.108 & 0.053 & 0.063 & 0.072 & 0.114 & 0.078 & 0.129 & 0.073 & 0.111 & 0.121 & 0.077 & 0.098 & 0.072 & 0.075 & 1.437 \\
\hline 0.142 & 0.158 & 0.066 & 0.115 & 0.114 & 0.126 & 0.111 & 0.149 & 0.105 & 0.164 & 0.135 & 0.151 & 0.154 & 0.127 & 0.137 & 0.125 & 0.088 & 2.166 \\
\hline 0.055 & 0.068 & 0.052 & 0.034 & 0.043 & 0.046 & 0.054 & 0.061 & 0.053 & 0.116 & 0.051 & 0.060 & 0.113 & 0.045 & 0.091 & 0.079 & 0.051 & 1.071 \\
\hline 0.063 & 0.094 & 0.049 & 0.051 & 0.032 & 0.053 & 0.100 & 0.092 & 0.052 & 0.095 & 0.064 & 0.070 & 0.080 & 0.036 & 0.045 & 0.066 & 0.048 & 1.091 \\
\hline 0.124 & 0.132 & 0.086 & 0.089 & 0.063 & 0.055 & 0.105 & 0.131 & 0.065 & 0.140 & 0.086 & 0.130 & 0.139 & 0.061 & 0.075 & 0.065 & 0.086 & 1.631 \\
\hline 0.108 & 0.120 & 0.084 & 0.070 & 0.086 & 0.101 & 0.062 & 0.115 & 0.059 & 0.135 & 0.083 & 0.107 & 0.127 & 0.061 & 0.070 & 0.109 & 0.057 & 1.554 \\
\hline 0.083 & 0.100 & 0.079 & 0.067 & 0.061 & 0.066 & 0.103 & 0.063 & 0.089 & 0.131 & 0.083 & 0.089 & 0.117 & 0.054 & 0.087 & 0.097 & 0.057 & 1.424 \\
\hline 0.146 & 0.157 & 0.123 & 0.111 & 0.080 & 0.130 & 0.141 & 0.155 & 0.063 & 0.175 & 0.111 & 0.144 & 0.160 & 0.114 & 0.130 & 0.133 & 0.075 & 2.148 \\
\hline 0.042 & 0.054 & 0.036 & 0.037 & 0.036 & 0.037 & 0.046 & 0.053 & 0.033 & 0.035 & 0.044 & 0.047 & 0.049 & 0.034 & 0.045 & 0.034 & 0.032 & 0.693 \\
\hline 0.108 & 0.120 & 0.070 & 0.059 & 0.081 & 0.105 & 0.079 & 0.102 & 0.054 & 0.085 & 0.046 & 0.076 & 0.100 & 0.065 & 0.061 & 0.087 & 0.039 & 1.337 \\
\hline 0.127 & 0.142 & 0.115 & 0.089 & 0.085 & 0.123 & 0.128 & 0.142 & 0.087 & 0.150 & 0.072 & 0.074 & 0.134 & 0.097 & 0.077 & 0.087 & 0.054 & 1.785 \\
\hline 0.070 & 0.075 & 0.075 & 0.066 & 0.059 & 0.058 & 0.098 & 0.072 & 0.052 & 0.124 & 0.086 & 0.097 & 0.062 & 0.102 & 0.109 & 0.088 & 0.068 & 1.361 \\
\hline 0.074 & 0.090 & 0.051 & 0.045 & 0.039 & 0.047 & 0.058 & 0.060 & 0.073 & 0.076 & 0.050 & 0.055 & 0.083 & 0.031 & 0.078 & 0.064 & 0.036 & 1.009 \\
\hline 0.085 & 0.093 & 0.070 & 0.076 & 0.052 & 0.060 & 0.090 & 0.106 & 0.060 & 0.115 & 0.056 & 0.107 & 0.111 & 0.054 & 0.047 & 0.061 & 0.047 & 1.292 \\
\hline 0.134 & 0.149 & 0.082 & 0.102 & 0.108 & 0.123 & 0.139 & 0.154 & 0.102 & 0.163 & 0.132 & 0.141 & 0.146 & 0.076 & 0.126 & 0.071 & 0.103 & 2.049 \\
\hline 0.144 & 0.141 & 0.077 & 0.115 & 0.121 & 0.129 & 0.140 & 0.155 & 0.107 & 0.170 & 0.122 & 0.145 & 0.141 & 0.109 & 0.122 & 0.118 & 0.054 & 2.109 \\
\hline 1.669 & 1.915 & 1.302 & 1.372 & 1.242 & 1.450 & 1.677 & 1.894 & 1.249 & 2.188 & 1.420 & 1.765 & 2.011 & 1.262 & 1.546 & 1.496 & 1.095 & \\
\hline
\end{tabular}

And finally, Table 6 shows the sequence of elements, which influence one another.

Table 6

The sequence of elements ( criteria )

\begin{tabular}{|c|c|c|c|c|c|c|c|}
\hline \multicolumn{2}{|c|}{$(\mathrm{J})$} & \multicolumn{2}{|c|}{$(\mathrm{R})$} & \multicolumn{2}{|c|}{$(\mathrm{R}+\mathrm{J})$} & \multicolumn{2}{|c|}{$(\mathrm{R}-\mathrm{J})$} \\
\hline 10 & 2.188 & 1 & 2.397 & 1 & 4.066 & 11 & 1.42 \\
\hline 13 & 2.011 & 3 & 2.166 & 12 & 3.55 & 17 & 1.014 \\
\hline 2 & 1.915 & 9 & 2.148 & 16 & 3.545 & 9 & 0.899 \\
\hline 8 & 1.894 & 17 & 2.109 & 3 & 3.468 & 3 & 0.864 \\
\hline 12 & 1.765 & 16 & 2.049 & 9 & 3.397 & 1 & 0.728 \\
\hline 7 & 1.677 & 12 & 1.785 & 13 & 3.372 & 16 & 0.553 \\
\hline 1 & 1.669 & 6 & 1.631 & 2 & 3.352 & 6 & 0.181 \\
\hline 15 & 1.546 & 7 & 1.554 & 8 & 3.318 & 12 & 0.02 \\
\hline 16 & 1.496 & 2 & 1.437 & 7 & 3.231 & 7 & -0.123 \\
\hline 6 & 1.450 & 8 & 1.424 & 17 & 3.204 & 5 & -0.151 \\
\hline 11 & 1.420 & 13 & 1.361 & 6 & 3.081 & 14 & -0.253 \\
\hline 4 & 1.372 & 11 & 1.337 & 10 & 2.881 & 15 & -0.254 \\
\hline 3 & 1.302 & 15 & 1.292 & 15 & 2.838 & 4 & -0.301 \\
\hline 14 & 1.262 & 5 & 1.091 & 11 & 2.757 & 8 & -0.47 \\
\hline 9 & 1.249 & 4 & 1.071 & 4 & 2.443 & 2 & -0.478 \\
\hline 5 & 1.242 & 14 & 1.009 & 5 & 2.333 & 13 & -0.65 \\
\hline 17 & 1.095 & 10 & 0.693 & 14 & 2.271 & 10 & -1.495 \\
\hline
\end{tabular}

Column (R-J) represents the position of an element (along the lateral axis) and the criteria whenever this number is positive, it indicates that criteria is influential, and in case it is negative, it means the criteria are influenced by other criteria. Also, $(\mathrm{R}+\mathrm{J})$ column indicates the total sum of the influence of an element (on the longitudinal axis) with regard to its influence and the influence exerted on it. Fig. 1 represents the position of the elements in the possible hierarchy.

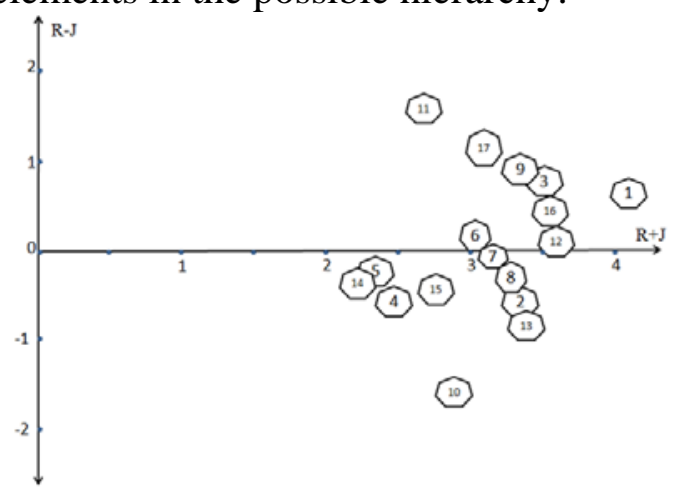

Fig. 1. Position of elements (criteria) 
As inferred from the results of Fig. 1, the criteria which along the horizontal axis of $(R+J)$ coordinate tend towards infinity are among criteria which according to the experts participating in this study have the highest effect on the other criteria in the area of Aluminum Composite Panel industry in Iran. According to Table 6, the weight of each criteria obtained with respect to interval ranking.

Table 7

The weight of criteria

\begin{tabular}{|c|c|c|c|}
\hline \multicolumn{2}{|c|}{ (R-J) } & \multicolumn{2}{|c|}{ Weight } \\
\hline 11 & 1.42 & 3.915 & 0.0891 \\
\hline 17 & 1.014 & 3.509 & 0.0799 \\
\hline 9 & 0.899 & 3.394 & 0.0773 \\
\hline 3 & 0.864 & 3.359 & 0.0765 \\
\hline 1 & 0.728 & 3.223 & 0.0734 \\
\hline 16 & 0.553 & 3.048 & 0.0694 \\
\hline 6 & 0.181 & 2.676 & 0.0609 \\
\hline 12 & 0.02 & 2.515 & 0.0573 \\
\hline 7 & -0.123 & 2.372 & 0.0540 \\
\hline 5 & -0.151 & 2.344 & 0.0534 \\
\hline 14 & -0.253 & 2.242 & 0.0510 \\
\hline 15 & -0.254 & 2.241 & 0.0510 \\
\hline 4 & -0.301 & 2.194 & 0.0500 \\
\hline 8 & -0.47 & 2.025 & 0.0461 \\
\hline 2 & -0.478 & 2.017 & 0.0459 \\
\hline 13 & -0.65 & 1.845 & 0.0420 \\
\hline 10 & -1.495 & 1 & 0.0228 \\
\hline Sum & & 43.9190 & 1.0000 \\
\hline
\end{tabular}

\subsection{Simple Additive Weighting (SAW)}

Simple Additive Weighting (SAW) is the oldest, most widely known and practically used method (Hwang \& Yoon, 1981; Chu et al., 2007; Ginevičius \& Podvezko 2008a, 2008b, 2008c; Ginevicius et al., 2008; Ginevičius \& Gineviciene, 2009; Zavadskas et al., 2007c; Jakimavicius \& Burinskiene, 2009; Podvezko et al., 2010; Sivilevicius et al., 2008). This technique obtains a weighted sum of the performance ratings of each alternative under all attributes. In the first step of this method, it scales the values of all attributes to make them comparable and eventually it sums up the values of the all attributes for each alternative (chou et al., 2008). SAW which is also referred as weighted linear combination or scoring methods or weighted sum method is a simple and most often applied multi attribute decision technique and it is based on the weighted average. An evaluation score is calculated for each alternative by multiplying the scaled value given to the alternative of that attribute with the weights of relative importance directly assigned by decision maker followed by summing of the products for all criteria. For numerical attributes scores are calculated by normalized values to match the standardized scale. The SAW is a comparable scale for all elements in the decision matrix, the comparable scale obtained by $\mathrm{r}_{\mathrm{ij}}$ for benefit criteria Eq. (5) and the worst criteria Eq. (6).

$$
\begin{aligned}
& V_{i j}=\frac{X_{i j}}{X_{j}^{\text {max }}} \\
& V_{i j}=\frac{X_{J}^{\text {min }}}{X_{i j}}
\end{aligned}
$$

The SAW method, underlying additive values function, computes as alternatives score $\mathrm{Vi}=\mathrm{V}(\mathrm{Ai})$ by adding weighting normalized values before eventually ranking alternatives.

$$
V_{i}=\sum_{j=1}^{m} W_{j} V_{i j}
$$

For $V \in R^{n * m}$ with $\mathrm{i}=\{1, \ldots \ldots, \mathrm{n}\}, \mathrm{j}=\{1 \ldots . . \mathrm{m}\} ; V_{i j}, W_{j} \in(0,1)$

Then the selected alternative, $A^{*}$ is: (Nancy, 2013) 
$A^{*}=\max _{i} \sum_{j} W_{j} V_{i j}$

Following the stepwise SAW method as mentioned above and by using seventeen criteria, ZB company wants to choose the best alternatives. These criteria have been mentioned in Table 3. The weight of criteria have been computed by using DEMATEL method as shown in Table 7. After preparing collected data from experts, based on values of very low, low, average, high, very high (scale values 19) and computed weight, we will start following steps of saw method :

\section{Table 8}

Decision matrix

\begin{tabular}{|c|c|c|c|c|c|c|c|c|c|c|c|c|c|c|c|c|c|c|}
\hline Criteria & Alternative & 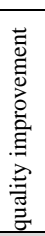 & 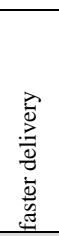 & 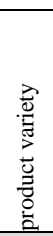 & 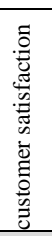 & 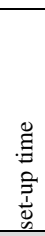 & 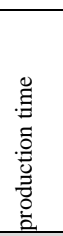 & 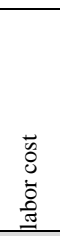 & 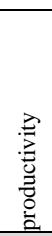 & 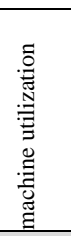 & 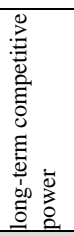 & 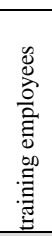 & 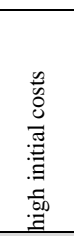 & 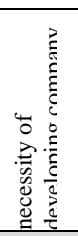 & 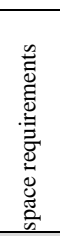 & 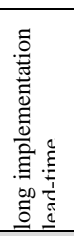 & 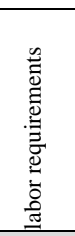 & 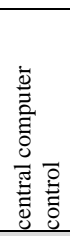 \\
\hline \multicolumn{2}{|c|}{ developing FMS } & 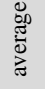 & 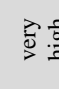 & $\stackrel{\sqrt{000}}{\nexists}$ & $\stackrel{\text { op }}{\stackrel{\text { of }}{I}}$ & $\underline{3}$ & $\stackrel{z}{a}$ & $\stackrel{3}{a}$ & 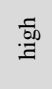 & 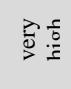 & $\stackrel{\bar{p}}{\mathrm{D}}$ & $\stackrel{3}{0}$ & 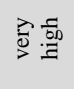 & 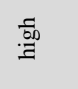 & $\stackrel{\sqrt[D]{0.0}}{=}$ & 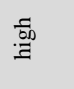 & 局 & $\underset{D}{2}$ \\
\hline \multicolumn{2}{|c|}{ Not developing FMS } & $\underline{\underline{z}}$ & 商 & $\stackrel{3}{0}$ & $\underline{z}$ & 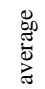 & 离 & 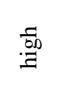 & 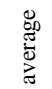 & 总 & 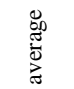 & 总 & 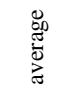 & $\underline{3}$ & 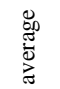 & $\underline{\underline{z}}$ & 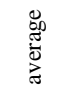 & 觉 \\
\hline
\end{tabular}

Table 9

Decision matrix (collected data based on scale values 1-9)

\begin{tabular}{|c|c|c|c|c|c|c|c|c|c|c|c|c|c|c|c|c|c|}
\hline Alternative & 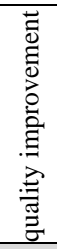 & 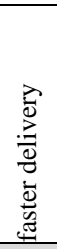 & 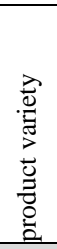 & 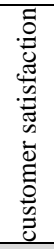 & 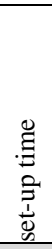 & 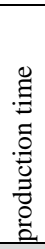 & 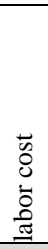 & 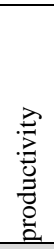 & 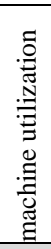 & 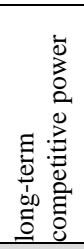 & 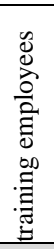 & 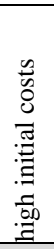 & 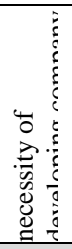 & 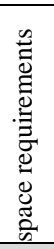 & 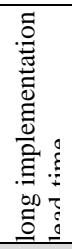 & 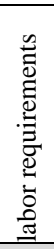 & 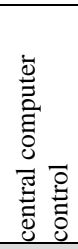 \\
\hline developing FMS & 5 & 9 & 7 & 7 & 7 & 7 & 7 & 7 & 9 & 9 & 7 & 5 & 7 & 3 & 3 & 9 & 9 \\
\hline Not developing FMS & 3 & 7 & 3 & 3 & 5 & 3 & 3 & 5 & 7 & 5 & 3 & 1 & 3 & 5 & 7 & 5 & 5 \\
\hline
\end{tabular}

Now calculate the normalized decision matrix according to positive criteria and negative and we finally find the ranking reported in Table 10.

\section{Table 11}

The Ranked Alternative

\begin{tabular}{ll}
\hline Implementing FMS & 0.7419 \\
Not implementing FMS & $\mathbf{0 . 7 8 8 2}$ \\
\hline
\end{tabular}

Finally according to SAW method, the implementation of FMS is not recommended.

\section{Conclusion}

So far, only a few Iranian companies have made a serious attempt to install FMS. This is mostly because FMSs are highly capital intensive and it might take several years to install them. ZB Company is one of the Iranian companies that have been successful in partial implementation of FMS. A hybrid model of DEMATEL and SAW was proposed by the authors of this paper to help management of ZB who could not make a decision about whether or not FMS should be implemented in the entire plant. Also, several important factors were found by the authors and the relative importance and influences of these factors on the objective of the decision-making model were investigated. The DEMATEL helped us 
incorporate 17 factors, which were both quantitative and qualitative to evaluate the implementation of FMS. Using SAW, it was concluded that implementation of FMSs was not recommended. Table 1 shows the ranking of alternative by DEMATEL and SAW methods. So, in implementing and developing of FMS, the top manager can concentrate on this strategic decision. This study revealed that DEMATEL and SAW could be used for managing complicated problems to assess advanced manufacturing technologies. Sensitivity analysis of results which is of great importance in practical decision-making, also using FUZZY DEMATEL for evaluation of manufacturing systems would make interesting subjects for future research.

\section{References}

Amiri, M., Sadaghiyani, J., Payani, N., \& Shafieezadeh, M. (2011). Developing a DEMATEL method to prioritize distribution centers in supply chain. Management Science Letters, 1(3), 279-288.

Bayazit, O. (2005). Use of AHP in decision-making for flexible manufacturing systems. Journal of Manufacturing Technology Management, 16(7), 808-819.

Boer, H., \& Krabbendam, K. (1991). The effective implementation and operation of flexible manufacturing systems. In achieving competitive edge getting ahead through technology and people (pp. 64-69). Springer London.

Boer, H., \& Krabbendam, K. (1992). Organizing for manufacturing innovation: the case of flexible manufacturing systems. International Journal of Operations \& Production Management, 12(7/8), 4156.

Chan, F. T., Kazerooni, A., \& Abhary, K. (1997). A fuzzy approach to operation selection. Engineering Applications of Artificial Intelligence, 10(4), 345-356.

Chan, F. T., Jiang, B., \& Tang, N. K. (2000). The development of intelligent decision support tools to aid the design of flexible manufacturing systems. International journal of production economics, 65(1), 7384.

Chiu, Y. J., Chen, H. C., Tzeng, G. H., \& Shyu, J. Z. (2006). Marketing strategy based on customer behaviour for the LCD-TV. International Journal of Management and Decision Making, 7(2), 143-165.

Chou, S. Y., Chang, Y. H., \& Shen, C. Y. (2008). A fuzzy simple additive weighting system under group decision-making for facility location selection with objective/subjective attributes. European Journal of Operational Research, 189(1), 132-145.

Chu, M. T., Shyu, J., Tzeng, G. H., \& Khosla, R. (2007). Comparison among three analytical methods for knowledge communities group-decision analysis. Expert systems with applications, 33(4), 1011-1024.

EVANS, G. W., \& HADDOCK, J. (1992). Modelling tools for flexible manufacturing systems. Production Planning \& Control, 3(2), 158-167.

Ginevičius, R., \& Ginevičiene, V. B. (2009). The compliance of master's degree studies with the economic needs of the country. Technological and Economic Development of Economy, 15(1), 136-153.

Ginevičius, R., \& Podvezko, V. (2008a). Multicriteria graphical-analytical evaluation of the financial state of construction enterprises. Technological and Economic Development of Economy, 14(4), 452-461.

Ginevičius, R., \& Podvezko, V. (2008b). Multicriteria evaluation of Lithuanian banks from the perspective of their reliability for clients. Journal of Business Economics and Management, 9(4), 257-267.

Ginevičius, R., \& Podvezko, V. (2008c). Housing in the context of economic and social development of Lithuanian regions. International Journal of Environment and Pollution, 35(2), 309-330.

Ginevičius, R., Podvezko, V., \& Andruškevičius, A. (2008). The effectiveness of diversification of construction enterprise activities. The 25th International Symposium on Automation and Robotics in Construction: selected papers, June 26-29. 2008 Vilnius, Lithuania, 759-763.

Hwang, C. L., \& Yoon, K. (1981). Multiple Attribute Decision Making: Methods and Applications: A State of the Art Survey. Sprinnger-Verlag, New York, NY.

Inman, R. A. (1991). Flexible manufacturing systems: issues and implementation. Industrial Management, 31(4), 7-11.

Jakimavičius, M., \& Burinskiene, M. (2009). A GIS and multi-criteria-based analysis and ranking of transportation zones of Vilnius city. Technological and Economic Development of Economy, 15(1), 3948. 
Kaighobadi, M., \& Venkatesh, K. (1994). Flexible manufacturing systems: an overview. International Journal of Operations \& Production Management, 14(4), 26-49.

Lin, K. M., \& Lin, C. W. (2008, October). Cognition map of experiential marketing strategy for hot spring hotels in Taiwan using the DEMATEL method. In Natural Computation, 2008. ICNC'08. Fourth International Conference on (Vol. 1, pp. 438-442). IEEE.

Lin, C. L., \& Tzeng, G. H. (2009). A value-created system of science (technology) park by using DEMATEL. Expert Systems with Applications, 36(6), 9683-9697.

Liou, J. J., Tzeng, G. H., \& Chang, H. C. (2007). Airline safety measurement using a hybrid model. Journal of Air Transport Management, 13(4), 243-249.

Maffei, M. J., \& Meredith, J. (1995). Infrastructure and flexible manufacturing technology: theory development. Journal of Operations Management, 13(4), 273-298.

Matta, A., Tolio, T., Karaesmen, F., \& Dallery, Y. (2001). An integrated approach for the configuration of automated manufacturing systems. Robotics and Computer-Integrated Manufacturing, 17(1), 19-26.

Mehregan, M., Razavi, S. M., \& Akhavan Anvari, M. R. (2012). Identification and Evaluation of Strategic Decisions in Gas Industry Using DEMATEL Method. Iranian Journal of Management Studies, 5(5), 4965.

Murat Albayrakoglu, M. (1996). Justification of new manufacturing technology: a strategic approach using the analytical hierarchy process. Production and Inventory Management Journal, 37, 71-76.

Myint, S., \& Tabucanon, M. T. (1994). A multiple-criteria approach to machine selection for flexible manufacturing systems. International Journal of Production Economics, 33(1), 121-131.

Nancy, S. B. (2013). Performance evaluation and comparison of MADM algorithms for subjective and objective weights in heterogeneous networks. International Journal of Emerging Trends in Electrical and Electronics (IJETEE), 2(2), 37-42.

Podvezko, V., Mitkus, S., \& Trinkūniene, E. (2010). Complex evaluation of contracts for construction. Journal of Civil Engineering and Management, 16(2), 287-297.

Rao, R. V. (2007). Decision Making in the Manufacturing Environment Using Graph Theory and Fuzzy Multiple Attribute Decision Making Methods. Springer.

Shamsuzzaman, M., Ullah, A. S., \& Bohez, E. L. (2003). Applying linguistic criteria in FMS selection: fuzzy-set-AHP approach. Integrated Manufacturing Systems, 14(3), 247-254.

Shang, J., \& Sueyoshi, T. (1995). A unified framework for the selection of a flexible manufacturing system. European Journal of Operational Research, 85(2), 297-315.

Shieh, J. I., Wu, H. H., \& Huang, K. K. (2010). A DEMATEL method in identifying key success factors of hospital service quality. Knowledge-Based Systems, 23(3), 277-282.

Sivilevicius, H., Zavadskas, E. K., \& Turskis, Z. (2008). Quality attributes and complex assessment methodology of the asphalt mixing plant. Baltic Journal of Road and Bridge Engineering, 3(3).

Solimanpur, M., Vrat, P., \& Shankar, R. (2005). An ant algorithm for the single row layout problem in flexible manufacturing systems. Computers \& Operations Research, 32(3), 583-598.

Tzeng, G. H., Chiang, C. H., \& Li, C. W. (2007). Evaluating intertwined effects in e-learning programs: A novel hybrid MCDM model based on factor analysis and DEMATEL. Expert Systems with Applications, 32(4), 1028-1044.

Tzeng, G. H., \& Huang, J. J. (2011). Multiple attribute decision making: methods and applications. CRC Press.

Wu, W. W., \& Lee, Y. T. (2007). Developing global managers' competencies using the fuzzy DEMATEL method. Expert Systems with Applications, 32(2), 499-507.

Yang, Y. P. O., Shieh, H. M., Leu, J. D., \& Tzeng, G. H. (2008). A novel hybrid MCDM model combined with DEMATEL and ANP with applications. International Journal of Operations Research, 5(3), 160168.

Yu, R., \& Tzeng, G. H. (2006). A soft computing method for multi-criteria decision making with dependence and feedback. Applied Mathematics and Computation, 180(1), 63-75.

Zavadskas, E. K., Turskis, Z., Dejus, T., \& Viteikiene, M. (2007). Sensitivity analysis of a simple additive weight method. International Journal of Management and Decision Making, 8(5), 555-574. 